\title{
Multifunctional antiperovskites driven by strong magnetostructural coupling
}

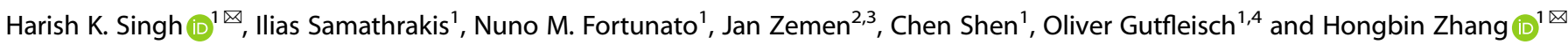

Based on density functional theory calculations, we elucidated the origin of multifunctional properties for cubic antiperovskites with noncollinear magnetic ground states, which can be attributed to strong isotropic and anisotropic magnetostructural coupling. Of 54 stable magnetic antiperovskites $\mathrm{M}_{3} \mathrm{XZ}(\mathrm{M}=\mathrm{Cr}, \mathrm{Mn}, \mathrm{Fe}, \mathrm{Co}$, and $\mathrm{Ni} ; \mathrm{X}=$ selected elements from Li to Bi except for noble gases and $4 f$ rare-earth metals; and $Z=C$ and $N$ ), 14 are found to exhibit the $\Gamma_{4 g} / \Gamma_{5 g}$ (i.e., characterized by irreducible representations) antiferromagnetic magnetic configurations driven by frustrated exchange coupling and strong magnetocrystalline anisotropy. Using the magnetic deformation as an effective proxy, the isotropic magnetostructural coupling is characterized, and it is observed that the paramagnetic state is critical to understand the experimentally observed negative thermal expansion and to predict the magnetocaloric performance. Moreover, the piezomagnetic and piezospintronic effects induced by biaxial strain are investigated. It is revealed that there is not a strong correlation between the induced magnetization and anomalous Hall conductivities by the imposed strain. Interestingly, the anomalous Hall/Nernst conductivities can be significantly tailored by the applied strain due to the fine-tuning of the Weyl points energies, leading to promising spintronic applications.

npj Computational Materials (2021)7:98; https://doi.org/10.1038/s41524-021-00566-w

\section{INTRODUCTION}

Smart materials like multiferroic materials with enhanced coupling between different degrees of freedom (e.g., mechanical, electronic, and magnetic) are promising for engineering devices for future applications such as sensors, transducers, memories, and spintronics $^{1-3}$. Cubic antiperovskite (APV) compounds host the two most appealing aspects of multiferroics, e.g., magnetoelectric coupling and piezomagnetic effect (PME) ${ }^{3,4}$. In APV materials, the strong magnetoelectric coupling is achieved by combining piezoelectric and piezomagnetic heterostructure composites ${ }^{5-7}$. A significant PME is reported for $\mathrm{Mn}$-based nitrides like $\mathrm{Mn}_{3} \mathrm{SnN}$, making such compounds a suitable component for fabricating magnetoelectric composite ${ }^{8,9}$. The PME in APVs can be attributed to the strong magnetostructural coupling, which manifests itself also as giant negative thermal expansion (NTE) ${ }^{10-12}$ and magnetocaloric/barocaloric effect ${ }^{9,13-17}$. From the materials perspective, many Mn-based APV carbides go through a first-order magnetic phase transition and possess a large magnetocaloric effect ${ }^{14,18}$. For instance, $\mathrm{Mn}_{3} \mathrm{GaC}$ exhibits a huge magnetic entropy change $\left(\Delta \mathrm{S}_{M}\right)$ of $15 \mathrm{~J} / \mathrm{kgK}$ under an applied magnetic field of $2 \mathrm{~T}^{16}$. The strong magnetostructural coupling in APVs is driven by the cubicto-cubic first-order transition wherein a change in the crystal volume brings about a change in the frustrated magnetic states. Last but not least, APVs have been investigated recently due to the presence of a treasury of multifunctionality such as superconductivity $^{19}$, thermoelectric ${ }^{20}$, magnetostriction ${ }^{21}$.

Particularly, from the topological transport properties point of view, the existence of finite anomalous Hall conductivity $(A H C)$ in noncollinear antiferromagnets has attracted noticeable attention due to possible applications in AFM spintronics for information storage and data processing ${ }^{22-25}$. The spin-dependent transport phenomena can provide spin-polarized charge current and large pure spin current, which could be achieved premised on two fundamental properties, i.e., AHC and spin Hall conductivity. The kagome lattice turns out to be an elementary model to host giant $\mathrm{AHC}^{26-28}$. Recently, Mn-based APV nitrides have been proposed to exhibit large $A H C$ in frustrated AFM kagome lattice ${ }^{29-32}$. It is observed that $\mathrm{Mn}_{3} \mathrm{GaN}$ exhibits vanishing and non-vanishing $\mathrm{AHC}$ for two different magnetic ordering $\Gamma_{5 g}$ and $\Gamma_{4 g}$, respectively ${ }^{29,30}$. In this regard, for magnetic materials with noncollinear AFM ground states, the non-vanishing $\mathrm{AHC}$ is only feasible with specific magnetic space group symmetry, i.e., the AHC tensor depends on the magnetic group symmetry ${ }^{33}$. For instance, $M_{3} X(X=G a, G e$, and $\mathrm{Sn})$ and $\mathrm{Mn}_{3} \mathrm{Z}(\mathrm{Z}=\mathrm{Ir}, \mathrm{Pt}$, and $\mathrm{Rh})$ have been reported to have a different form of $\mathrm{AHC}$ tensor as a result of different magnetic ordering ${ }^{22,34-36}$. A possible phase transition $\left(\Gamma_{5 g} \leftrightarrow \Gamma_{4 g}\right)$ attained by strain or chemical modification could make these materials suitable for AFM spintronic applications. The spin-polarized current could also be generated by temperature gradient instead of the applied electric fields, resulting in anomalous Nernst conductivity (ANC), also termed as spin caloritronics ${ }^{37,38}$. A large ANC in noncollinear AFMs could be useful for establishing spin caloritronics devices that exhibit useful prospects in energy conversion and information processing. Noticeably, a large ANC of $1.80 \mathrm{AK}^{-1} \mathrm{~m}^{-1}$ has been reported for $\mathrm{APV} \mathrm{Mn}_{3} \mathrm{NiN}$ at $200 \mathrm{~K}^{39}$, which is slightly less than half of the highest reported ANC of 4.0 $\mathrm{AK}^{-1} \mathrm{~m}^{-1}$ in $\mathrm{CO}_{2} \mathrm{MnGa}^{40,41}$. Enhancing the AHC and ANC by applying strain could be crucial for realizing AFM spintronic devices.

In this work, we carried out a systematic analysis of 54 cubic APV systems $(P m \overline{3} \mathrm{~m})$ with chemical formula $M_{3} X Z$ (see Supplementary Fig. 1) to determine their magnetic ground states, tunability via biaxial strain, and the resulting spintronic properties (Fig. 1). The selected 54 cubic APVs are stable, i.e., fulfilling all three stability criteria such as thermodynamical, mechanical, and dynamical stabilities, which were recently evaluated in our

\footnotetext{
${ }^{1}$ Institute of Materials Science, Technische Universität Darmstadt, Darmstadt, Germany. ${ }^{2}$ Faculty of Electrical Engineering, Czech Technical University, Prague, Czech Republic. ${ }^{3}$ Department of Physics, Blackett Laboratory, Imperial College London, London, UK. ${ }^{4}$ Materials Recycling and Resource Strategies, Fraunhofer IWKS, Hanau, Germany.

凶email: harish@tmm.tu-darmstadt.de; hzhang@tmm.tu-darmstadt.de
} 


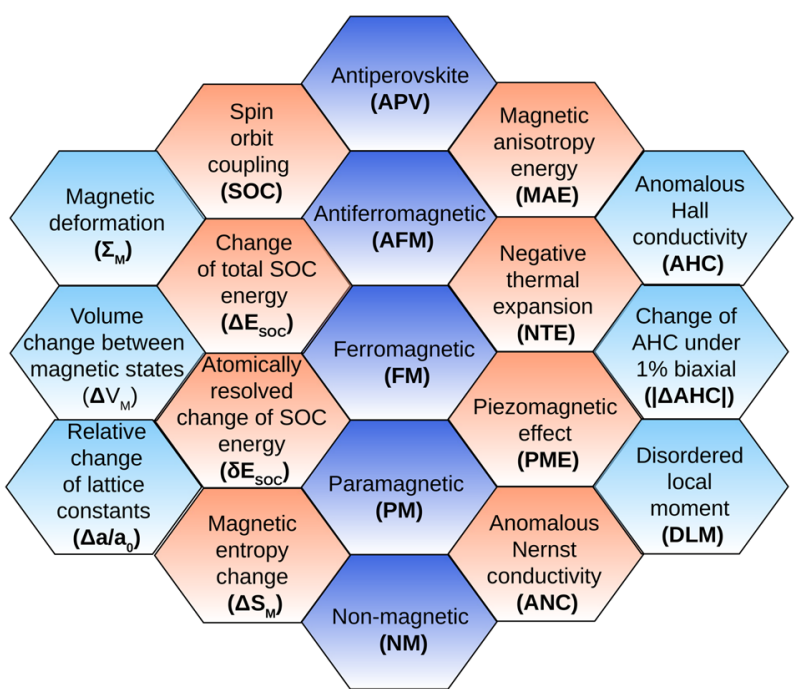

Fig. 1 Abbreviations. The list of abbreviations used in the manuscript.

high-throughput screening ${ }^{42}$. Explicit calculations were performed to obtain the energies of eight phases, i.e., $\Gamma_{4 g}, \Gamma_{5 g}$, non-magnetic (NM), ferromagnetic (FM), collinear AFM-1 (CAFM-1), collinear AFM2 (CAFM-2), collinear AFM-3 (CAFM-3), and M-1 configurations. Moreover, the magnetic anisotropy energy (MAE) defined as the energy difference between $\Gamma_{5 g}$ and $\Gamma_{4 g}$ is examined to understand the origin of the noncollinear magnetic states with the help of spin-orbit coupling (SOC) energy. Moreover, a detailed analysis of the lattice constant variation with respect to the magnetic states reveals that the paramagnetic (PM) state is critical in the magnetic phase transition, enabling us to predict potential NTE and magnetocaloric materials. Last but not least, the PME was studied by introducing biaxial strains (compressive and tensile), which causes possible phase transitions between $\Gamma_{5 g} \leftrightarrow \Gamma_{4 g}$, and leads to a significant modification in the $\mathrm{AHC}$ and $\mathrm{ANC}$, dubbed as a piezospintronic effect ${ }^{30,43}$. In-depth analysis on symmetry analysis and electronic structure suggests that the piezospintronic effect is originated from the existence of Weyl nodes whose position can be tailored by strain, resulting in promising applications for future spintronic devices.

\section{RESULTS}

\section{Validation and prediction of magnetic ground state}

As the magnetic transition metal atoms are located at the face centers of the cubic cell for magnetic APVs, it leads to a frustrated kagome lattice in the (111)-plane. Correspondingly, noncollinear magnetic structures are expected when the interatomic exchange interaction is AFM for the nearest neighbors. As shown in Fig. 2, $\Gamma_{4 g}$ and $\Gamma_{5 g}$ are the two most common magnetic configurations reported for APVs, resulting in $120^{\circ}$ magnetic angle configurations within the (111)-plane between three magnetic moments. The $\Gamma_{4 g}$ state can be obtained from $\Gamma_{5 g}$ by simultaneously rotating the moments of three metal atoms within the (111)-plane by $90^{\circ}$. The APVs with the noncollinear magnetic ground state are listed in Table 1, in comparison to the available experimentally measured results. Interestingly, all the APVs with noncollinear magnetic ground states are nitrides including $\mathrm{Cr}$ and $\mathrm{Mn}$, while all carbides end up with the FM and CAFM-2 ground state except $\mathrm{Mn}_{3} \mathrm{SnC}$, which exhibits the $\Gamma_{5 g}$ state (see Supplementary Table 1).

$\mathrm{Mn}_{3} \mathrm{GaC}^{44}$ and $\mathrm{Mn}_{3} \mathrm{Ga}_{0.95} \mathrm{~N}_{0.94}{ }^{15}$ are reported to adopt collinear AFM and M-1 magnetic configurations, respectively. Thus, we considered additionally CAFM-1, CAFM-2, CAFM-3, and M-1 configurations (see Fig. 2e-h). It is confirmed that $\mathrm{Mn}_{3} \mathrm{GaC}$ exhibit the CAFM-2 magnetic ground state, the same as $M n_{3} A I C$ and $\mathrm{Mn}_{3} \mathrm{InC}$. This is in contrast to the previous experimental reports on both $\mathrm{Mn}_{3} \mathrm{AlC}^{45}$ and $\mathrm{Mn}_{3} \mathrm{InC}^{46}$ with a FM configuration. Moreover, $\mathrm{Cr}_{3} \mathrm{SnN}$ and $\mathrm{Mn}_{3} \mathrm{SnN}$ exhibit the cAFM-3 magnetic ground state, where it is demonstrated that $\mathrm{Mn}_{3} \mathrm{SnN}$ shows four different magnetic and crystallographic phases (see Supplementary Note $1)^{47,48}$. Such discrepancies might be attributed to the temperature effect, indicating a strong interplay between magnetism and crystal structures at finite temperatures that deserves further detailed investigations. Interestingly, none of the APV is stabilized in the $M-1$ phase (see Supplementary Table 1). It is noted that there are also other magnetic configurations such as ferrimagnetic and canted states for $M n_{3} X C(X=S n$ and $Z n)$, which will be saved for future investigations.

The magnetic ground states of noncollinear APVs are in good agreement with the experimental measurements. For instance, both $\mathrm{Mn}_{3} \mathrm{GaN}^{13}$ and $\mathrm{Mn}_{3} \mathrm{ZnN}^{47,49}$ have the $\Gamma_{5 g}$ magnetic ground state, which are consistent with our density functional theory (DFT) calculations. Interestingly, many Mn-based APVs exhibit a mixed $\Gamma_{4 g}+\Gamma_{5 g}$ magnetic ordering, with a possible metamagnetic transition to the other magnetic phases ${ }^{47}$. For instance, $\mathrm{Mn}_{3} \mathrm{AgN}$ is characterized by two distinct magnetic phase transitions. A mixed $\Gamma_{4 g}+\Gamma_{5 g}$ phase exists below $55 \mathrm{~K}$, whereas pure $\Gamma_{5 g}$ state persists at intermediate temperature range $(55 \mathrm{~K}<$ $\mathrm{T}<290 \mathrm{~K}$ ), and lastly, it undergoes the magnetic transition to PM state at $290 \mathrm{~K}^{47}$. Likewise, $\mathrm{Mn}_{3} \mathrm{NiN}$ displays such mixed magnetic phases $^{47}$.

The mixed magnetic ordering can be attributed to the MAE between the $\Gamma_{5 g}$ and $\Gamma_{4 g}$ states, which can be expressed as:

$\mathrm{MAE}=\mathrm{E}_{\Gamma_{5 g}}-\mathrm{E}_{\Gamma_{4 g}}$,

where $\mathrm{E}$ indicates the total energy of the corresponding magnetic configuration. For $\mathrm{Mn}_{3} \mathrm{AgN}$ and $\mathrm{Mn}_{3} \mathrm{NiN}$ with experimentally observed mixed magnetic states, the corresponding MAE is 0.063 and $0.144 \mathrm{meV}$, respectively. Nevertheless, it is still unclear why $\mathrm{Mn}_{3} \mathrm{AgN}$ is stabilized in the $\Gamma_{5 g}$ state between 55 and $290 \mathrm{~K}$. We suspect that those compounds with MAE greater than $0.2 \mathrm{meV}$ between $\Gamma_{4 g}$ and $\Gamma_{5 g}$ should have pure noncollinear magnetic states. In our study, the Mn-based APVs $\mathrm{Mn}_{3} \mathrm{XN}$ with $\mathrm{X}=\mathrm{Ag}, \mathrm{Co}, \mathrm{Ir}$, $\mathrm{Ni}, \mathrm{Pd}$, and Rh exhibit $\Gamma_{4 g}$ while those with $\mathrm{X}=\mathrm{Au}, \mathrm{Ga}, \mathrm{Hg}$, In, Pt, and $Z n$ display the $\Gamma_{5 g}$ as the magnetic ground state (see Table 1 and Supplementary Table 1). This is consistent with the recent DFT calculations for $\mathrm{Mn}_{3} \mathrm{XN}(\mathrm{X}=\mathrm{Ni}, \mathrm{Zn}, \mathrm{Ga} \text {, and } \mathrm{Pt})^{50}$, except for $\mathrm{Mn}_{3} \mathrm{XN}\left(\mathrm{X}=\mathrm{In}, \mathrm{Pd}\right.$, and Ir). In ref. ${ }^{50}, \mathrm{Mn}_{3} \operatorname{lnN}$ exhibit $\Gamma_{4 g}$ state while $\mathrm{Mn}_{3} \mathrm{XN}(\mathrm{X}=\mathrm{Ir}$ and $\mathrm{Pd})$ display $\Gamma_{5 g}$ state. The MAE for $\mathrm{Mn}_{3} \mathrm{InN}$ calculated by Huyen et al. is $74.6 \mathrm{meV}$, which is much larger than our MAE (-1.84 meV). As detailed below, a large MAE could be expected for the compound with strong SOC; thus, it is questionable for $\mathrm{Mn}_{3} \mathrm{InN}$ with such a large MAE of $74.6 \mathrm{meV}^{50}$. To clarify the discrepancy, we performed calculations of the MAE using the Quantum Espresso (QE) $\operatorname{code}^{51}$ with the same parameters consider in Huyen et al. study. The QE and VASP calculations provide the same magnetic ground state and MAE for $\mathrm{Mn}_{3} \mathrm{XN}(\mathrm{X}=\mathrm{Ir}$ and $\mathrm{Pt}$ ) systems, which is in contradiction to the Huyen study performed using $\mathrm{QE}^{50}$.

Lastly, based on our high-throughput calculations ${ }^{42}$, two unreported $\mathrm{Cr}$-based APVs are stable, our calculations reveal that $\mathrm{Cr}_{3} \mathrm{IrN}$ and $\mathrm{Cr}_{3} \mathrm{PtN}$ have $\Gamma_{4 g}$ as the lowest energy magnetic configuration (see Table 1 and Supplementary Table 1). While among APV nitrides, $\mathrm{Mn}_{3} \mathrm{AuN}$ and $\mathrm{Mn}_{3} \mathrm{HgN}$ are the other two unreported noncollinear systems with $\Gamma_{5 g}$ magnetic ground state. To the best of our knowledge, there is no experimental neutron diffraction measurement available till now for such APVs, making them interesting for future studies. To shed more light on the origin of MAE, we performed detailed calculations of the SOC energy in order to obtain atomically resolved contributions to the $M A E$. Like MAE, the change in the total SOC energy $\left(\triangle \mathrm{E}_{\mathrm{SOC}}\right)$ of $\mathrm{M}_{3} \mathrm{XN}$ can be defined as the difference between the SOC energy 


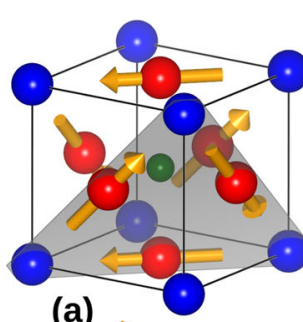

(a)

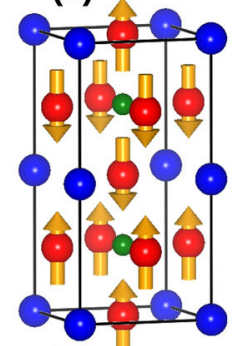

(e)

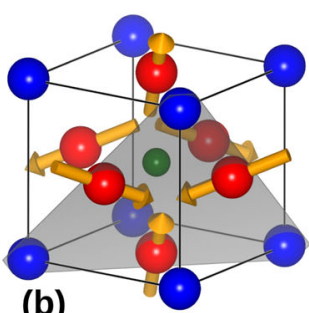

(b)

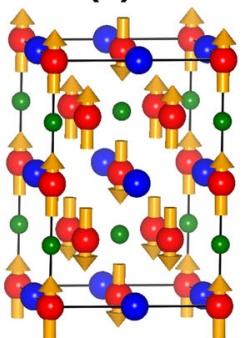

(f)

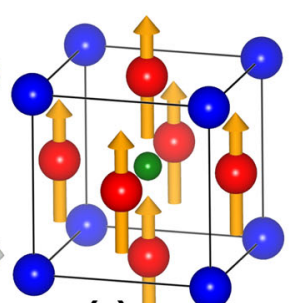

(c)

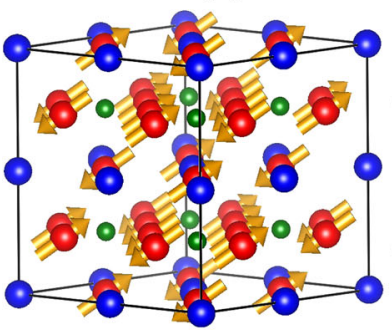

(g)

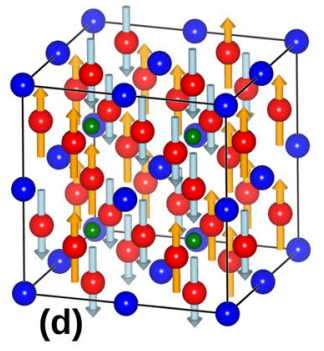

(d)

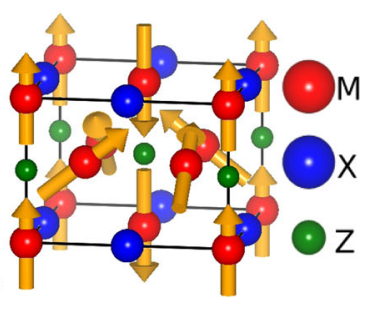

(h)

Fig. 2 Magnetic configurations. Possible magnetic structures of antiperovskites: a $\Gamma_{5 g}, \mathbf{b} \Gamma_{4 g}, \mathbf{c}$ FM, d PM, e cAFM-1, f cAFM-2, g cAFM-3, and h $\mathrm{M}-1$. The spin chirality for $\Gamma_{4 g}$ and $\Gamma_{5 g}$ is assumed to be +1 .

Table 1. Compilation of the APVs $\left(M_{3} X Z\right)$ with noncollinear magnetic ground state.

\begin{tabular}{|c|c|c|c|c|c|c|}
\hline$M_{3} X Z$ & magGS & ${ }^{\mathrm{M}}\left(\delta \mathrm{E}_{\mathrm{SOC}}\right)$ & ${ }^{x}\left(\delta \mathrm{E}_{\mathrm{SOC}}\right)$ & $\Delta \mathrm{E}_{\mathrm{SOC}}$ & MAE & Refs. \\
\hline $\mathrm{Cr}_{3} \mathrm{IrN}$ & $\Gamma_{4 g}$ & -0.367 & 4.532 & 4.164 & 2.187 & \\
\hline $\mathrm{Cr}_{3} \mathrm{PtN}$ & $\Gamma_{4 g}$ & -0.936 & 5.639 & 4.703 & 2.868 & \\
\hline $\mathrm{Mn}_{3} \mathrm{AgN}$ & $\Gamma_{4 g}$ & 0.3987 & -0.2486 & 0.151 & 0.063 & $\begin{array}{l}\Gamma_{4 g}+\Gamma_{5 g} \\
\text { ref. } 47\end{array}$ \\
\hline $\mathrm{Mn}_{3} \mathrm{AuN}$ & $\Gamma_{5 g}$ & 1.205 & -4.353 & -3.148 & -1.671 & \\
\hline $\mathrm{Mn}_{3} \mathrm{CoN}$ & $\Gamma_{4 g}$ & 0.005 & 1.716 & 1.721 & 1.218 & AFM ref. ${ }^{11}$ \\
\hline $\mathrm{Mn}_{3} \mathrm{GaN}$ & $\Gamma_{5 g}$ & -1.111 & 1.10 & -1.110 & -0.291 & $\begin{array}{l}\Gamma_{5 g} \\
\text { ref. }\end{array}$ \\
\hline $\mathrm{Mn}_{3} \mathrm{HgN}$ & $\Gamma_{5 g}$ & -0.746 & -3.691 & -4.438 & -1.551 & \\
\hline $\mathrm{Mn}_{3} \ln \mathrm{N}$ & $\Gamma_{5 g}$ & -0.734 & -0.224 & -0.958 & -1.849 & \\
\hline $\mathrm{Mn}_{3} \mathrm{IrN}$ & $\Gamma_{4 g}$ & 0.599 & 19.705 & 20.305 & 10.548 & \\
\hline $\mathrm{Mn}_{3} \mathrm{NiN}$ & $\Gamma_{4 g}$ & 1.216 & -0.87 & 0.346 & 0.144 & $\begin{array}{l}\Gamma_{4 g}+\Gamma_{5 g} \\
\text { ref. }\end{array}$ \\
\hline $\mathrm{Mn}_{3} \mathrm{PdN}$ & $\Gamma_{4 g}$ & 1.292 & -1.115 & 0.176 & 0.357 & AFM ref. ${ }^{11}$ \\
\hline $\mathrm{Mn}_{3} \mathrm{PtN}$ & $\Gamma_{5 g}$ & 3.872 & -13.877 & -10.005 & -4.969 & \\
\hline $\mathrm{Mn}_{3} \mathrm{RhN}$ & $\Gamma_{4 g}$ & -0.021 & 3.482 & 3.460 & 1.702 & \\
\hline $\mathrm{Mn}_{3} \mathrm{ZnN}$ & $\Gamma_{5 g}$ & -0.756 & -0.105 & -0.861 & -1.452 & $\begin{array}{l}\Gamma_{5 g} \\
\text { refs. } 47,49\end{array}$ \\
\hline
\end{tabular}

All the units are in meV. For each compound, the calculated magnetic ground state (magGS) is listed and the change in the atomically resolved SOC energy ( $\delta \mathrm{E}_{\mathrm{SOC}}$ ) between $\Gamma_{5 g}$ and $\Gamma_{4 g}$ state is enumerated for $\mathrm{M}$ and $\mathrm{X}$ atoms. The calculated MAE and $\Delta \mathrm{E}_{\mathrm{SOC}}$ are summarized as given by Eqs (1) and (2), respectively. The last column lists the available experimental magnetic ground state.

$\left(\mathrm{E}_{\mathrm{SOC}}\right)$ of the $\Gamma_{5 g}$ and $\Gamma_{4 g}$ magnetic states ${ }^{52}$.

$\Delta \mathrm{E}_{\mathrm{SOC}}=\mathrm{E}_{\mathrm{SOC}}\left(\Gamma_{5 g}\right)-\mathrm{E}_{\mathrm{SOC}}\left(\Gamma_{4 g}\right)$,

In the same way, the atomically resolved change of SOC energy $\left(\delta \mathrm{E}_{\mathrm{soc}}\right)$ can also be defined for each atomic species, as shown in Table 1 and Supplementary Table 3. The positive (negative) values of MAE and $\Delta \mathrm{E}_{\mathrm{SOC}}$ indicate the $\Gamma_{4 g}\left(\Gamma_{5 g}\right)$ magnetic ground state. On the one hand, it is found that the sign of MAE is the same as that of $\Delta \mathrm{E}_{\mathrm{SOC}}$ (see Table 1). That is, both the $\Delta \mathrm{E}_{\mathrm{SOC}}$ and MAE are equally valid to characterize the magnetic ground states, with a linear scaling behavior observed (see Supplementary Fig. 2). For example, $\mathrm{Cr}_{3} \mathrm{PtN}$ has $\Gamma_{4 g}$ magnetic ground state with an MAE of $2.868 \mathrm{meV}$ and $\Delta \mathrm{E}_{\mathrm{SOC}}$ of $4.70 \mathrm{meV}$. The atomic resolved $\delta \mathrm{E}_{\mathrm{SOC}}$ of $\mathrm{Pt}$ and $\mathrm{Cr}$ are 5.639 and $-0.938 \mathrm{meV}$. Evidently, the $\delta \mathrm{E}_{\mathrm{SOC}}$ of $\mathrm{Pt}$ is larger than $\mathrm{Cr} \delta \mathrm{E}_{\mathrm{SOC}}$ originating in the strong $\mathrm{SOC}$ of Pt. As a result, the $\delta \mathrm{E}_{\mathrm{SOC}}$ of $\mathrm{Pt}$ is a determining factor for the MAE and $\Delta \mathrm{E}_{\mathrm{SOC}}$ sign. On the other hand, for most APVs, the contribution of the $X$ elements to MAE is more significant than that of the magnetic elements $M$, as indicated by the atomic resolved $\delta E_{\text {soc }}$ (see Table 1). Taking the $\mathrm{Mn}_{3} \mathrm{XN}$ with $\mathrm{X}=\mathrm{Co}, \mathrm{Rh}$, and Ir as examples, the MAE are 1.22, 1.70, and $10.55 \mathrm{meV}$, corresponding to the dominant $\delta \mathrm{E}_{\mathrm{SOC}}$ of $\mathrm{X}$ as $1.72,3.46$, and $20.31 \mathrm{meV}$, respectively. It is noted that $\delta E_{\mathrm{SOC}}$ of $\mathrm{Mn}$ is smaller than $3 \%$ of $\Delta E_{\mathrm{SOC}}$ for such compounds. This can be attributed to the enhanced strength of atomic SOC of the $\mathrm{X}$ elements, e.g., the strength of atomic SOC for Co, Rh, and Ir atoms is about 0.065, 0.152, and $0.452 \mathrm{eV}$, respectively ${ }^{53}$. Therefore, the enhanced atomic SOC strength of the $\mathrm{X}$ elements is favorable for the strong MAE of the APV compounds.

\section{NTE and barocaloric}

Turning now to the magnetostructural coupling, which can be best represented for APVs by the NTE, magnetocaloric, and PME. NTE materials exhibit contraction in the lattice parameters with respect to temperature in contrast to most materials. ${ }^{49}$ To study NTE of APVs, we evaluated the relative change in the lattice constants $\Delta \mathrm{a}$ / $\mathrm{a}_{0}$ on the transition from the noncollinear magnetic ground state to PM state (where $a_{0}$ is the lattice constant of $\Gamma_{4 g}$ or $\Gamma_{5 g}$ noncollinear state and $\Delta \mathrm{a}$ is lattice constant difference between PM and $\Gamma_{4 g}$ or $\Gamma_{5 g}$ state) and compared with the $\Delta a / a_{0}$ values obtained from the experimental measurement at their transition temperature (see Fig. $3 a)^{11,12,49}$. The average of the evaluated $\Delta a / a_{0}$ is in good agreement with the experimental observation except for $\mathrm{Mn}_{3} \mathrm{GaN}$ (see 3a). Together with the mismatch for the other cases, we suspect it may be due to the finite temperature effect, as our DFT calculations are done at zero Kelvin.

Importantly, it is found that the PM state is critical and cannot be approximated as the NM state, e.g., the $\Delta a / a_{0}$ between the noncollinear and NM states is about five times as large as the experimental value (see Fig. 3a). An ameliorated estimation could be achieved by defining the PM phase as a collinear AFM arrangement generated by using the disordered local moment (DLM) theory ${ }^{54}$. The $\Delta \mathrm{a} / \mathrm{a}_{0}$ of $\mathrm{Cr}_{3} \mathrm{IrN}$ and $\mathrm{Cr}_{3} \mathrm{PtN}$ on the transition 

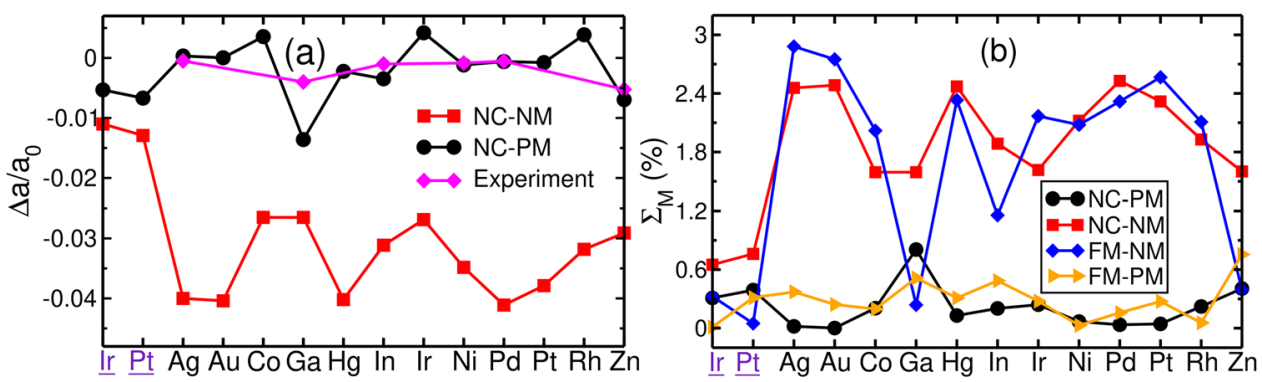

Fig. 3 Negative thermal expansion and barocaloric. a The relative change in the lattice constant $\left(\Delta a / a_{0}\right)$ and $\mathbf{b}$ the magnetic deformation $\Sigma_{M}$ for the $\mathrm{Cr}_{3} \mathrm{X} \mathrm{N}$ and $\mathrm{Mn}_{3} \mathrm{XN}$ antiperovskites (X and $\mathrm{X}$ are the elements present on the $\mathrm{X}$-axis in violet and black, respectively). The lattice constants of noncollinear, FM, PM, and NM states are used to determine $\Delta a / a_{0}$ and $\Sigma_{M}$.

from $\Gamma_{4 g}$ magnetic ground state to PM are -0.0053 and -0.0067 , respectively. That is, Cr-based APVs undergo the lattice contraction with $\Delta \mathrm{a} / \mathrm{a}_{0}$ comparable to the reported NTE in Mn-based APV materials such as $\mathrm{Mn}_{3} \mathrm{GaN}$ and $\mathrm{Mn}_{3} \mathrm{ZnN}$ (see Fig. 3a). Furthermore, the equilibrium lattice constants decrease and increase for the $\mathrm{Mn}_{3} \mathrm{XN}(\mathrm{X}=\mathrm{Ga}, \mathrm{Hg}, \mathrm{In}, \mathrm{Ni}, \mathrm{Pd}, \mathrm{Pt}$, and $\mathrm{Zn})$ and $\mathrm{Mn}_{3} \mathrm{XN}$ APVs $(X=A u, C o, I r$, and $R h$ ) on the transition from a noncollinear magnetic ground state to PM state, respectively (see Fig. 3a).

Recently, Bocarsly et al. proposed a magnetic deformation proxy $\Sigma_{M}$ to predict the magnetocaloric materials as the theoretical evaluation of $\left(\Delta S_{M}\right)$ is very challenging ${ }^{55}$. This proxy measures the volume deformation of the NM and magnetic structure, which correlates well with the experimentally measured $\Delta S_{M}$ values. In their study, they screened 167 FM materials, including 7 APVs. For the APVs, it is noted that $\Sigma_{M}$ scales linearly with the percentage volume difference $\Delta V_{M}$ between the FM and NM unit cell (see Supplementary Fig. 3). Inspired by their work, we evaluated $\Sigma_{M}$ for the APVs with FM and noncollinear magnetic ground state in order to estimate the potential magnetocaloric effect. For FM APVs, it is found that the $\Sigma_{M}$ is most significant for the Fe-based APVs within the 1.2-1.7\% range (see Supplementary Table 5). For example, the $\Sigma_{M}$ of $\mathrm{Fe}_{3} \mathrm{RhN}, \mathrm{Fe}_{3} \mathrm{PtN}$, and $\mathrm{Fe}_{3} \mathrm{NiN}$ are $1.67 \%, 1.59 \%$, and $1.54 \%$, respectively, whereas Co-, $\mathrm{Mn}-$, and Ni-based APVs have $\Sigma_{M}<1.0 \%$ except for $\mathrm{Mn}_{3} \mathrm{ZnC}(1.33 \%)$ (see Supplementary Table 5). Thus, we expect a significant magnetocaloric effect in $\mathrm{Fe}_{3} \mathrm{RhN}, \mathrm{Fe}_{3} \mathrm{PtN}$, and $\mathrm{Fe}_{3} \mathrm{NiN}$, based on the argument that the $\Sigma_{M}$ approximate to $1.5 \%$ could exhibit a considerable magnetocaloric effect ${ }^{55}$.

As to the noncollinear APVs, we evaluated the $\Sigma_{M}$ by considering four $\Delta \mathrm{V}_{M}$ combinations, namely, the noncollinear magnetic ground state with PM and NM states (NC-PM and NCNM), the FM with PM and NM states (FM-PM and FM-NM) (see Fig. $3 \mathrm{~b}$ and Supplementary Table 6). It is observed that the $\Sigma_{M}$ value is small by using the $\Delta \mathrm{V}_{M}$ of the NC-PM and FM-PM states, in comparison to the other two combinations (see Fig. 3b). Nevertheless, a small value of $\Sigma_{M}$ does not mean a low $\Delta \mathrm{S}_{M}{ }^{55}$. For instance, the experimentally measured $\Delta \mathrm{S}_{M}$ of $\mathrm{Mn}_{3} \mathrm{GaN}$ is very significant $\left(22.3 \mathrm{Jkg}^{-1} \mathrm{~K}^{-1}\right)$, and it also exhibits giant barocaloric effect ${ }^{13}$. Overall, the $\Sigma_{M}$ of $\mathrm{Mn}_{3} \mathrm{GaN}$ should be largest among all the APVs. The calculated $\Sigma_{M}$ of $\mathrm{Mn}_{3} \mathrm{GaN}$ is largest $0.805 \%$ for the $\Gamma_{5 g}$ and PM state combination, which verifies the experimental observation, whereas the $\Sigma_{M}$ of $\mathrm{Mn}_{3} \mathrm{GaN}$ for the other three combinations is not the largest among the APVs (see Fig. 3b). Therefore, it can be concluded for the noncollinear systems that the approach of Bocarsly et al. proxy does not provide reasonable $\Sigma_{M}$ obtained from the $\Delta \mathrm{V}_{M}$ combination of NC-NM, FM-NM, and FM-PM states. The implicit correlation of $\Delta S_{M}$ with $\Sigma_{M}$ indicates the strong magnetostructural coupling in APVs with significant $\Delta \mathrm{V}_{M}$ (NC-PM). This conduces to the prediction of possible NTE and magnetocaloric materials with sizable $\Sigma_{M}$ by virtue of significant $\Delta \mathrm{V}_{M}$ (see Supplementary Table 6). For example, the $\mathrm{Cr}_{3} \mathrm{XN}$ APVs $(\mathrm{X}=\mathrm{Ir}$ and $\mathrm{Pt})$ with appreciable $\Sigma_{M}$ could exhibit potential applications as NTE and magnetocaloric materials.

\section{Piezomagnetism}

PME provides another effective characterization of the magnetostructural coupling ${ }^{6}$, which manifests itself as the response of magnetization to strain. The origin of PME is explained based on symmetry together with an illustration of the magnetic spin directions (see Supplementary Fig. 4 and Supplementary Note 2). For APVs with noncollinear magnetic ground states, the total bulk magnetization is vanishing, but a net magnetization can be induced under finite compressive/tensile strain, as reported for $\mathrm{Mn}$-based $\mathrm{APVs}^{6,8}$. Our calculations confirm their results on the Mn-based APVs except for the $\mathrm{Mn}_{3} \mathrm{CoN}$ and $\mathrm{Mn}_{3} \mathrm{RhN}$ (see Supplementary Fig. 5), where the resulting net magnetization from our (Zemen et al.) $)^{8}$ calculations are $0.646(0.305)$ and 0.214 $(-0.143) \mu_{B} / f . u$., respectively. Interestingly, for $\mathrm{Mn}_{3} \mathrm{CoN}$, a net magnetic moment of $0.362 \mu_{B} / f . u$. is induced at Co atoms under $1 \%$ tensile strain, whereas the local magnetic moment of Co is zero in the cubic unstrained state. The magnetic moment direction of $\mathrm{Co}$ is aligned in (111) plane similar to $\mathrm{Mn}$ atom direction in the $\Gamma_{4 g}$ state. As a result, $\mathrm{Mn}_{3} \mathrm{CoN}$ could be an interesting material for elastocaloric and magneto-elastic applications. Surprisingly, the PME effect is asymmetric with respect to the applied compressive and tensile strains (see Supplementary Figs. 6 and 7). Most APVs exhibit more significant PME with tensile strain except $\mathrm{Cr}_{3} \mathrm{PtN}$ (see Supplementary Fig. 6), e.g., the net magnetization for $\mathrm{Cr}_{3} \mathrm{PtN}$ at $1 \%$ compressive strain is as large as $0.21 \mu_{\mathrm{B}} /$ f.u. in comparison to $0.15 \mu_{\mathrm{B}} /$ f.u. at $1.0 \%$ tensile strain.

The magnitude of the magnetoelectric effect is minimal in the intrinsic bulk AFM materials, such as $\mathrm{Cr}_{2} \mathrm{O}_{3}{ }^{56}$. The two-phase heterostructure materials consolidate the magnetoelectric effect. A recent study corroborates APV as a potential material for magnetoelectric composite ${ }^{6,57}$. The piezoelectric perovskite could be one suitable substrate for such composite heterostructure as they also have comparable lattice parameters with $\mathrm{APVs}^{5,58}$. The heterostructure amalgamate the piezoelectric and piezomagnetic properties, which are coupled by an interfacial strain. Based on our PME analysis, we propose $\mathrm{Cr}$ - and $\mathrm{Mn}$-based noncollinear systems with significant PME as potentials candidates for magnetoelectric composite.

Interestingly, the biaxial strain can induce phase transition between different magnetic configurations, i.e., $\Gamma_{4 g}$ and $\Gamma_{5 g}$, which can further lead to a significant change in the transport properties as discussed below. It is found that a few APV materials undergo a magnetic phase transition due to the imposed biaxial strain (see Supplementary Tables 4 and 7). For instance, cubic $M n_{3} A u N$ has $\Gamma_{5 g}$ as the magnetic ground state, which preserves under tensile strain. However, a transition into a distorted $\Gamma_{4 g}$ state is obtained by applying a compressive strain of 0.5 and $1.0 \%$ (see Supplementary Table 4). Such transitions can be attributed to significant changes in the MAE and with respect to the biaxial strain (see Supplementary Table 4), but there is no consistent MAE trend as the absolute value of MAE is mostly determined by the atomic SOC strengths. 


\section{Anomalous Hall conductivity (unstrained)}

It is well known that FM compounds commonly exhibit AHC due to the presence of a net magnetization ${ }^{59}$, where the broken timereversal symmetry $(\mathcal{T})$ and $\mathrm{SOC}$ are two essential prerequisites. Noncollinear AFMs also display non-zero AHC, as observed in $\mathrm{Mn}_{3} \mathrm{X}(\mathrm{X}=\mathrm{Ir}, \mathrm{Ge} \text {, and } \mathrm{Sn})^{22,34}$. As a linear response property, the occurrence of AHC can be elucidated based on the symmetry analysis. For cubic APVs, the magnetic space group of $\Gamma_{5 g}$ and $\Gamma_{4 g}$ are $R \overline{3} m$ (166.97) and $R \overline{3} m^{\prime}$ (166.101), respectively. For $\Gamma_{5 g}$, the local magnetic moment spin directions of $M$ atoms are invariant under the mirror symmetry transformation $M_{(0 \overline{1} 1)}, M_{(10 \overline{1})}$, and $M_{(\overline{1} 10)}$, leading to vanishing $\mathrm{AHC}$ (see Supplementary Table 8 ) $^{29,30}$, whereas in the $\Gamma_{4 g}$ state, the above-mentioned mirror symmetries are broken, but the product of mirror $(M)$ and time-reversal symmetry $\mathcal{T}$ retain the $\Gamma_{4 g}$ configuration. This results in finite AHCs with all three off-diagonal components non-zero but of the same amplitude (see Supplementary Table 8$)^{29}$.

Explicit calculations confirm the above symmetry arguments. For instance, the $\mathrm{AHC}$ is zero for $\mathrm{Mn}_{3} \mathrm{XN}(\mathrm{X}=\mathrm{Au}, \mathrm{Hg}, \mathrm{In}, \mathrm{Pt}$, and $\mathrm{Zn})$ with the $\Gamma_{5 g}$ ground state (see Supplementary Figs. 8b and 9), whereas the significant AHC is observed for the APVs with the $\Gamma_{4 g}$ ground state, such as the $\mathrm{AHC}$ of $\mathrm{Cr}_{3} \mathrm{IrN}$ and $\mathrm{Cr}_{3} \mathrm{PtN}$ are 414.6 and $278.6 \mathrm{~S} / \mathrm{cm}$, respectively. The AHC of APVs is summarized in the Supplementary information (see Supplementary Figs. 8-10 and 16). In comparison to the previous studies on an individual or a few compounds ${ }^{29,32}$, our results are in good agreement (see Supplementary Table 9). For instance, the $\mathrm{AHC}$ of $\mathrm{Mn}_{3} \mathrm{SnN}$ from our and calculations by Gurung et al. are 106.5 and $133 \mathrm{~S} / \mathrm{cm}^{29}$, respectively, whereas the value $(-73.9 \mathrm{~S} / \mathrm{cm})$ obtained by Huyen et al. $^{50}$ is of opposite sign in addition to the difference in the absolute values. In this regard, for $\mathrm{Mn}_{3} \mathrm{NiN}$, the sign of experimentally observed AHC is confirmed by our DFT calculations $^{31}$, consistent with that obtained by Zhou et al $^{32,39}$. This might be due to opposite magnetization direction while maintaining the noncollinear configurations or chirality of noncollinear spin configurations specified in the calculations, subject to further investigations. Moreover, it is observed that the absolute values of $\mathrm{AHC}$ for the same compound in the same magnetic ground state are scattered as well (see Supplementary Table 9). We suspect that this is due to the different lattice constants and numerical parameters used in different calculations. For instance, Zhou et al. performed calculations using the experimental lattice constants on $\mathrm{Mn}_{3} \mathrm{NiN}$ and other APV compounds, which are on average about $1.0-1.3 \%$ smaller than the fully relaxed lattice constants in this work.

\section{Strain-induced AHC (piezospintronics)}

As discussed above, all cubic APVs with noncollinear magnetic ground states display significant PMEs where a net magnetization can be induced by applying biaxial strain; an interesting question is whether the AHC can be tailored as well. From the symmetry point of view, for the $\Gamma_{5 g}$ state, the magnetic space group changes from $R \overline{3} m$ (166.97) to $C 2 / m$ (12.58) with biaxial strain, resulting in finite $\sigma_{x}$ and $\sigma_{y}$ with the same amplitude but opposite sign, while the $\sigma_{z}$ component remains zero ${ }^{30}$. This is confirmed by our calculations on those compounds with the $\Gamma_{5 g}$ ground state. For instance, the $\mathrm{AHC}$ induced by $1 \%$ biaxial strain is as large as $-71.2 \mathrm{~S} / \mathrm{cm}$ for $\mathrm{Mn}_{3} \mathrm{PtN}$ (see Supplementary Fig. 8b), comparable to that in $\mathrm{Mn}_{3} \mathrm{HgN}$ (see Supplementary Fig. 9). The $\mathrm{Mn}_{3} \operatorname{lnN}$ and $\mathrm{Mn}_{3} \mathrm{ZnN}$ have quite low AHC due to weak SOC strength (see Supplementary Fig. 9).

Similarly, the AHC of APVs in the $\Gamma_{4 g}$ state can also be tailored by the biaxial strain. In this case, the magnetic space group is reduced from $R \overline{3} m^{\prime}$ (166.101) to $C 2^{\prime} / m^{\prime}(12.62)$, the resulting $\sigma_{x}$ and $\sigma_{y}$ components are the same while the $\sigma_{z}$ component possesses a distinct value (see Supplementary Fig. 8a and Supplementary

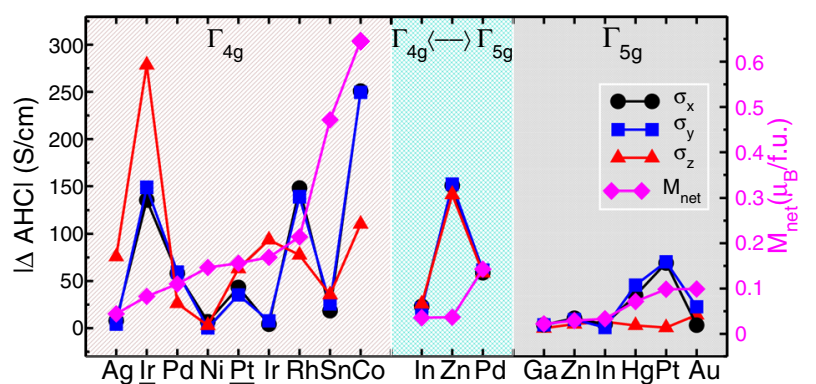

Fig. 4 Piezospintronic. The piezospintronic effect is illustrated by the change in $\mathrm{AHC}(|\Delta \mathrm{AHC}|)$ at $1 \%$ tensile strain and cubic phase. The $|\triangle \mathrm{AHC}|$ and net magnetization is shown for the $\mathrm{Cr}_{3} \mathrm{X} \mathrm{N}$ and $\mathrm{Mn}_{3} \mathrm{XN}$ antiperovskites ( $\mathrm{X}$ and $\mathrm{X}$ are the elements present on the $x$-axis, respectively). The left, middle, and right panels correspond to the $\Gamma_{4 g}$, phase transition between $\Gamma_{4 g}$ and $\Gamma_{5 g}$, and $\Gamma_{5 g}$ state, respectively.

Table 8). Explicit evaluations of the AHC for APVs in distorted $\Gamma_{4 g}$ states verify the symmetry arguments, where the $\sigma_{x}$ and $\sigma_{y}$ components behave the same with respect to strain (see Supplementary Fig. 8a). Interestingly, it is observed that more significant changes occur in AHC for the $\Gamma_{4 g}$ compounds than the $\Gamma_{5 g}$ cases. For instance, the $\sigma_{z}$ component of $\mathrm{AHC}$ in $\mathrm{Cr}_{3} \mathrm{IrN}$ attains the largest $A H C$ of $693.1 \mathrm{~S} / \mathrm{cm}$ at $1 \%$ tensile strain, with an increase of $278 \mathrm{~S} / \mathrm{cm}$ compared to the value in the cubic geometry (see Supplementary Fig. 8a). Moreover, the moderate strain can even lead to a sign change of the $\mathrm{AHC}$, as observed on the $\sigma_{z}$ component of $\mathrm{Mn}_{3} \mathrm{AgN}$ and $\mathrm{Mn}_{3} \mathrm{CoN}$ with $0.5 \%$ compressive and $1 \%$ tensile strain, respectively (see Supplementary Fig. 10).

One interesting question is whether the piezospintronic effect (i.e., the variation of $\mathrm{AHC}$ induced by biaxial strain) correlates with the PME, as summarized in Fig. 4 for the variation of such quantities comparing the cases with $1 \%$ tensile strain and cubic geometries. Obviously, $|\triangle A H C|$ cannot be directly inferred from the induced net magnetization, particularly exemplified by the APVs in the $\Gamma_{4 g}$ magnetic ground state. For example, the $|\triangle A H C|$ and net magnetization of $\mathrm{Cr}_{3} \mathrm{lrN}$ are $278.5 \mathrm{~S} / \mathrm{cm}\left(\sigma_{z}\right.$ component) and $0.082 \mu_{B} / f$.u., respectively, while for $\mathrm{Mn}_{3} \mathrm{SnN}$, the $|\triangle \mathrm{AHC}|$ and net magnetization are $35.5 \mathrm{~S} / \mathrm{cm}\left(\sigma_{z}\right.$ component $)$ and $0.47 \mu_{\mathrm{B}} /$ f.u., respectively. Moreover, for $\mathrm{Mn}_{3} \mathrm{CoN}$, the net magnetization and $|\triangle \mathrm{AHC}|$ of $\sigma_{x}$ are $250.7 \mathrm{~S} / \mathrm{cm}$ and $0.646 \mu_{\mathrm{B}} / \mathrm{f}$.u. It signifies that the $|\triangle \mathrm{AHC}|$ could be large for the small net magnetization and vice versa (see Fig. 4). Interestingly, the $|\triangle \mathrm{AHC}|$ and net magnetization are smaller for those compounds in the $\Gamma_{5 g}$ magnetic state, in comparison to those of such materials with the $\Gamma_{4 g}$ magnetic state, e.g., the $|\triangle A H C|$ and net magnetization of $M_{3} Z \mathrm{ZnN}$ are $9.17 \mathrm{~S} / \mathrm{cm}$ and $0.029 \mu_{\mathrm{B}} /$ f.u., respectively.

Particularly, the APVs undergoing a phase transition between $\Gamma_{4 g}$ and $\Gamma_{5 g}$ states driven by the epitaxial strain exhibit strong piezospintronic effect while the magnitude of the PME is marginal. For instance, $\mathrm{Mn}_{3} \mathrm{ZnN}$ has a $\Gamma_{5 g}$ magnetic ground state in the cubic phase with vanishing AHC. A phase transition into the $\Gamma_{4 g}$ state by $1 \%$ tensile strain results in a considerable $|\triangle A H C|$ of $152.2 \mathrm{~S} / \mathrm{cm}$ for the $\sigma_{y}$ component (see Fig. 4). On the other hand, the phase transition from the $\Gamma_{4 g}$ to $\Gamma_{5 g}$ states for $\mathrm{Mn}_{3} \mathrm{PdN}$ leads to a $|\triangle \mathrm{AHC}|$ as large as $58.6 \mathrm{~S} / \mathrm{cm}$ (Fig. 4). Therefore, we suspect that those compounds with $\Gamma_{4 g}$ states are more promising to host a strong piezospintronic effect.

\section{Anomalous Nernst effect}

The intriguing behavior of AHC under biaxial strain can be attributed to the fine-tuning of the electronic structure. To further illustrate such sensitivity, we investigated the ANC, which shares the same symmetry as $A H C$ but is proportional to the derivative of $\mathrm{AHC}$ with respect to energy following the Mott formula ${ }^{60}$. Taking 


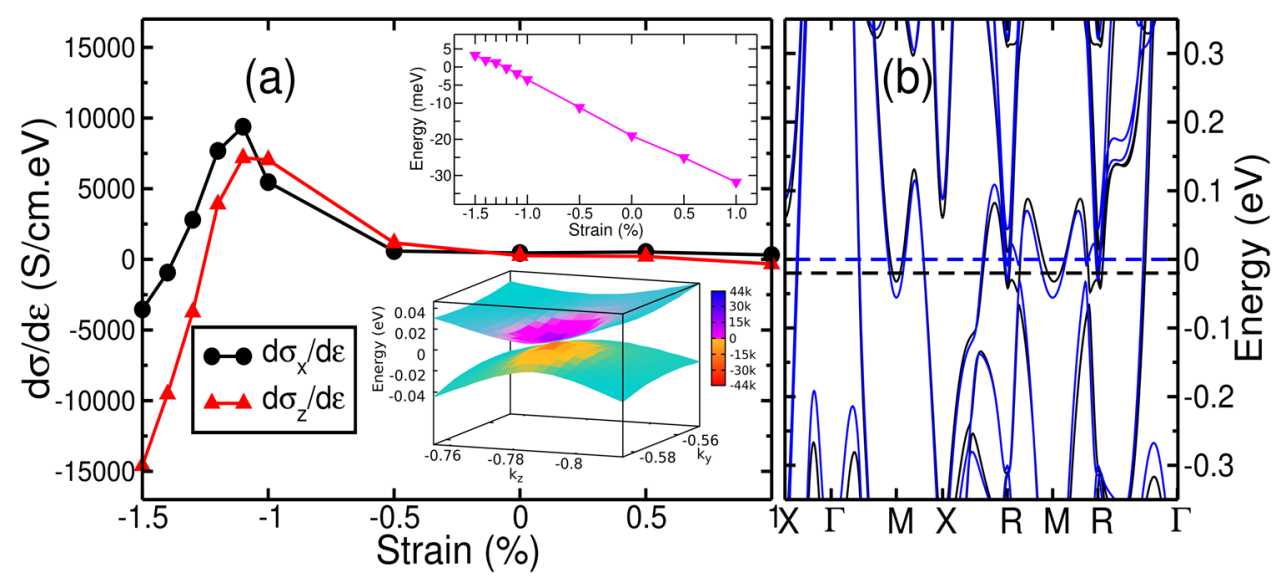

Fig. 5 Anomalous Nernst effect. a The calculated ANC of $\mathrm{Mn}_{3} \mathrm{PdN}$ in the $\Gamma_{4 g}$ magnetic state. The Weyl points $(0.43,-0.35,-0.49$, and equivalent coordinates) shift across the Fermi level with strain (inset above) and the Berry curvature for the $1.4 \%$ compressive biaxial strain (inset below). $\mathbf{b}$ The band structure of $\mathrm{Mn}_{3} \mathrm{PdN}$ for unstrained (black) and $1.4 \%$ compressive biaxial strained (blue). The presence of Weyl point near the Fermi energy is indicated by black (cubic) and blue dashed line ( $-1.4 \%$ biaxial strain).

$\mathrm{Mn}_{3} \mathrm{NiN}$ as an example, the corresponding $\mathrm{ANC}$ is as large as $16,569 \mathrm{~S} / \mathrm{cm} . \mathrm{eV}$. This is consistent with $1.80 \mathrm{AK}^{-1} \mathrm{~m}^{-1}$ at $200 \mathrm{~K}$ obtained by Zhou et al. ${ }^{39}$, up to a factor of two but with alike dependence with respect to the energy around the Fermi energy (see Supplementary Fig. 15). The numerical discrepancy might be due to the different lattice constants used in the calculations, as discussed above. Such a large ANC of $\mathrm{Mn}_{3} \mathrm{NiN}$ is comparable to the largest values observed experimentally in $\mathrm{CO}_{2} \mathrm{FeGe}$ $\left(3.16 \mathrm{AK}^{-1} \mathrm{~m}^{-1}\right), \quad \mathrm{Co}_{2} \mathrm{MnGa} \quad\left(4.0 \mathrm{AK}^{-1} \mathrm{~m}^{-1}\right)$, and $\mathrm{Fe}_{3} \mathrm{Ga}$ $\left(3.0 \mathrm{AK}^{-1} \mathrm{~m}^{-1}\right)^{40,41,61,62}$. Interestingly, the strain also has a stronger influence on the ANC. For the tetragonally distorted $\mathrm{Mn}_{3} \mathrm{NiN}$ in the $\Gamma_{4 g}$ state, the ANC becomes as large as $20,035 \mathrm{~S} / \mathrm{cm} . e V$ at $-0.5 \%$ compressive strain for the $a_{x}$ and $a_{y}$ components, i.e., enhanced by $21 \%$ compared to that of the cubic case. Last but not least, a sign change can be induced in the ANC by the biaxial strain. For instance, the ANC corresponding to the $a_{x}$ and $a_{y}$ components of $\mathrm{Cr}_{3} \mathrm{IrN}$ changes to $-3196.9 \mathrm{~S} / \mathrm{cm} . \mathrm{eV}$ at $1 \%$ compressive strain starting from $6823.4 \mathrm{~S} / \mathrm{cm} . \mathrm{eV}$ for the cubic case in the $\Gamma_{4 g}$ state. This is also observed for $\mathrm{Mn}_{3} \mathrm{AgN}$ and $\mathrm{Mn}_{3} \mathrm{CoN}$ (see Supplementary Fig. 11). The ANC of APV compounds as a function of biaxial strain is summarized in Supplementary Figs. 11-14.

To explicate the origin of the piezospintronic effects on both $\mathrm{AHC}$ and ANC, our detailed analysis of the electronic structure reveals that the tunability of $\mathrm{AHC}$ and ANC by strain can be attributed to the presence of Weyl points close to the Fermi energy. Taking $\mathrm{Mn}_{3} \mathrm{PdN}$ as an example, as shown in Fig. $5 \mathrm{a}$, the ANC can be tuned between $7037.3 \mathrm{~S} / \mathrm{cm} . \mathrm{eV}$ at $1 \%$ compressive strain to $-14,599 \mathrm{~S} / \mathrm{cm} . \mathrm{eV}$ at $1.5 \%$ compressive strain, with a sign change at $1.3 \%$ strain. Such an ANC is comparable to that observed in $\mathrm{Mn}_{3} \mathrm{NiN}$, awaiting further experimental validation. The band structure shows no obvious changes due to the applied strain (see Fig. 5b). However, it is found that there are 12 Weyl points at $(0.43,-0.35$, and -0.49$)$ and equivalent k-points with an energy of $20 \mathrm{meV}$ below the Fermi energy in the cubic phase. Such Weyl points will be shifted across the Fermi energy upon applying compressive strain, e.g., reaches to $-1.75 \mathrm{meV}$ for $-1.3 \%$ biaxial strain, and $1.87 \mathrm{meV}$ above the Fermi energy for $1.4 \%$ strain (see above inset of Fig. 5a). The AHC is mostly enhanced when the Weyl points are located at the Fermi energy, due to the singular behavior of the Berry curvature at the Weyl nodes (see below inset of Fig. 5a). This is consistent with ref. ${ }^{50}$ and our observation in $\mathrm{Mn}_{3} \mathrm{GaN}^{30,50}$. For magnetic materials, the Weyl nodes with opposite chiralities (i.e., Berry curvatures with opposite sign) are located at the same energy, but the total contributions to the AHC can be significant, particularly if the Weyl nodes are within "several" or "a few" meV around the Fermi energy.
Correspondingly, the sign of ANC, which is determined by the derivative of AHC can be tuned as the Weyl points are shifted across the Fermi level. We suspect such tunability of AHC and concomitant ANC by manipulating the Weyl points with the biaxial strain or other possible stimuli is promising for future applications, such as $\mathrm{Fe}_{3} \mathrm{Al}$ with giant transversal thermoelectric effects $^{62}$.

\section{DISCUSSION}

We carried out a systematic analysis of 14 cubic APV $M_{3} X Z$ compounds with noncollinear magnetic ground states, focusing on the magnetic properties driven by isotropic and anisotropic magnetostructural coupling. It is found that there exists a strong competition between different noncollinear magnetic configurations where a large MAE clearly defines the noncollinear magnetic ground state, while small MAE leads to the mixed $\Gamma_{4 g}$ and $\Gamma_{5 g}$ configurations. For such materials with mixed magnetic ions, the MAE cannot be understood based on the perturbation theory, whereas the SOC energy can be used to get a reliable atomic resolved contribution, which is mostly determined by the strength of atomic SOC. The magnetic ground state analysis resulted in the prediction of four unreported APVs with noncollinear ground state, especially $\mathrm{Cr}_{3} \mathrm{XN}$ APVs $\left(\mathrm{X}=\mathrm{Ir}\right.$ and $\mathrm{Pt}$ ) with the $\Gamma_{4 q}$ magnetic configuration. The isotropic magnetostructural coupling indicated by the magnetic deformation $\Delta \mathrm{a} / \mathrm{a}_{0}$ can be considered as an effective descriptor for the magnetocaloric effect. However, we observed that the magnetic deformation is better measured comparing the PM and magnetically ordered states, resulting in better agreement with the experimental NTE results, rather than comparing the NM and ordered state. Therefore, we suggest that the recently proposed proxy for predicting magnetocaloric effects based on the magnetic deformation could be improved by using quasi-random approximation of the PM state. More interestingly, biaxial strain not only causes a significant PME in such materials but also leads to a strong influence on MAE, AHC, and ANC after considering SOC. Based on detailed symmetry analysis, we performed an explicit evaluation of the AHC and found that those compounds with the $\Gamma_{4 g}$ magnetic ground state have large AHC, which is susceptible to the epitaxial strain. Nevertheless, there is no strong correlation between the net magnetization and induced $\mathrm{AHC}$ when finite strain is applied. Detailed analysis reveals that the sensitivity of $A H C$ and derived ANC with respect to strain can be attributed to the fine-tuning of energies for the Weyl nodes, opening up further possibilities for engineering spintronic devices in the future. 


\section{METHODS}

\section{Density functional theory calculation}

Our DFT calculations are performed using the projector augmented wave method as effectuated in the VASP package ${ }^{63}$. The exchange-correlation functional is approximated using the generalized gradient approximation as parameterized by Perdew-Burke-Ernzerhof ${ }^{64}$. We used an energy cutoff of $500 \mathrm{eV}$ for the plane-wave basis set, and a uniform k-points grid of $13 \times 13 \times 13$ within the Monkhorst-pack scheme for the Brillouin zone integrations. The Methfessel-Paxton scheme is used to determine the partial occupancies of orbitals with a smearing width of $0.06 \mathrm{eV}$. The SOC is considered in all the calculations. In order to verify the magnetic ground state of various compounds, the more accurate total energy calculations are performed using a higher energy cutoff of $600 \mathrm{eV}$ and a dense k-mesh of $25 \times 25 \times 25$ (see Supplementary Note 3 ).

The magnetic ground states are obtained by comparing the total energies (see Supplementary Table 1) of eight magnetic configurations, i.e., $\Gamma_{4 g}, \Gamma_{5 g}$, NM, FM, CAFM-1, CAFM-2, CAFM-3, and M-1 states (see Fig. 2), where the lattice constants are fully optimized for $\Gamma_{4 g}, \Gamma_{5 g}, \mathrm{FM}, \mathrm{PM}$, and NM state (see Supplementary Table 2). To verify the effects of the Coulomb interaction, we performed a series of DFT $+\mathrm{U}(\mathrm{U}=2-10 \mathrm{eV}$ for the $\mathrm{Mn}-3 d$ orbitals) calculations on $\mathrm{Mn}_{3} \mathrm{GaN}$. It is observed that the $\Gamma_{5 g}$ magnetic ground state does not change with respect to the applied $\mathrm{U}$ values (see Supplementary Table 10). Therefore, we suspect that the Coulomb interaction is likely to have a marginal influence on the magnetic ground state of such a class of compounds. Moreover, to investigate the piezomagnetic and piezospintronic effects, biaxial in-plane strain is applied, which reduces the crystalline symmetry from cubic to tetragonal. The optimal lattice constants along c-direction are evaluated by the polynomial fitting of the energies from a series of calculations.

\section{Modeling of paramagnetic state}

The PM state is modeled based on the DLM picture (see Fig. 2d), where a special quasi-random structure modeled using a $2 \times 2 \times 2$ supercell by imposing zero total magnetization ${ }^{65}$. That is, a supercell with the same number of up and down moments is considered with a pair correlation function same as $A_{50} B_{50}$ random alloys, generated using the alloy theoretic automated toolkit code ${ }^{66}$. It is noted that our results reveal that systematic treatment of the PM state is needed in order to provide a quantitative description of the magnetostructural coupling in such compounds, where the recently developed spin-lattice dynamics approach is promising for future detailed investigations ${ }^{67}$.

\section{Anomalous Hall and Nernst conductivity calculation}

The AHC is evaluated using the WannierTools code ${ }^{68}$, where the required accurate tight-binding models are obtained by the maximally localized Wannier functions (MLWFs) using the Wannier90 code ${ }^{69}$. The $s, p$, and $d$ orbitals of $\mathrm{M}$ and $\mathrm{X}$ atoms and the $\mathrm{s}$ and $\mathrm{p}$ orbitals for $\mathrm{N}$ atom are considered, resulting in $80 \mathrm{MLWFs}$ in total for every noncollinear APVs. The AHC is computed by integrating the Berry curvature on a uniform $401 \times 401 \times 401$ k-points mesh to guarantee good accuracy, which can be expressed as ${ }^{70}$ :

$\sigma_{x y}=-\frac{e^{2}}{\hbar} \int \frac{d \mathbf{k}}{(2 \pi)^{3}} \sum_{n} f[\epsilon(\mathbf{k})-\mu] \Omega_{n, x y}(\mathbf{k})$

$\Omega_{n, x y}(\mathbf{k})=-2 \operatorname{lm} \sum_{m \neq n} \frac{\left\langle\psi_{\mathbf{k} n}\left|v_{x}\right| \psi_{\mathbf{k} m}\right\rangle\left\langle\psi_{\mathbf{k} m}\left|v_{y}\right| \psi_{\mathbf{k} n}\right\rangle}{\left[\epsilon_{m}(\mathbf{k})-\epsilon_{n}(\mathbf{k})\right]^{2}}$

where e is elementary charge, $\mu$ is the chemical potential, $\psi_{n / m}$ denotes the Bloch wave function with energy eigenvalue $\epsilon_{n / m}, v_{x / y}$ is the velocity operator along Cartesian $x / y$ direction, and $f[\epsilon(\mathbf{k})-\mu]$ is Fermi-Dirac distribution function. The ANC $a_{x y}$ is evaluated based on the Mott relation, which yields:

$a_{x y}=-\left.\frac{\pi^{2} k_{B}^{2} T}{3 e} \frac{d \sigma_{x y}}{d \epsilon}\right|_{\epsilon=\mu}$

In this work, we evaluated only the derivative of AHC at the Fermi level $\mathrm{d} \sigma_{x y} / \mathrm{d} \epsilon$, which provides a quantitative measurements of the ANC.

\section{DATA AVAILABILITY}

All relevant computational results generated or analyzed during this study are included in the manuscript and Supplementary information files in the form of tables and figures.

\section{CODE AVAILABILITY}

The AHC was calculated using open source code Wannier90 and WannierTools. Postprocessing scripts used to calculate the derivative of AHC can be provided on request from the corresponding authors.

Received: 23 September 2020; Accepted: 3 June 2021; Published online: 30 June 2021

\section{REFERENCES}

1. Fiebig, M., Lottermoser, T., Meier, D. \& Trassin, M. The evolution of multiferroics. Nat. Rev. Mater. 1, 16046-16059 (2016).

2. Trassin, M. Low energy consumption spintronics using multiferroic heterostructures. J. Phys. Condens. Matter 28, 033001-033016 (2015).

3. Ma, J., Hu, J., Li, Z. \& Nan, C.-W. Recent progress in multiferroic magnetoelectric composites: from bulk to thin films. Adv. Mater. 23, 1062-1087 (2011).

4. Spaldin, N. A. \& Fiebig, M. The renaissance of magnetoelectric multiferroics. Science 309, 391-392 (2005).

5. Quintela, C. X. et al. Epitaxial antiperovskite/perovskite heterostructures for materials design. Sci. Adv. 6, eaba4017-eaba4023 (2020).

6. Lukashev, P., Sabirianov, R. F. \& Belashchenko, K. Theory of the piezomagnetic effect in mn-based antiperovskites. Phys. Rev. B 78, 184414-184418 (2008).

7. Shao, D.-F., Gurung, G., Paudel, T. R. \& Tsymbal, E. Y. Electrically reversible magnetization at the antiperovskite/perovskite interface. Phys. Rev. Mater. 3, 024405-024413 (2019).

8. Zemen, J., Gercsi, Z. \& Sandeman, K. G. Piezomagnetism as a counterpart of the magnetovolume effect in magnetically frustrated $\mathrm{mn}$-based antiperovskite nitrides. Phys. Rev. B 96, 024451-024458 (2017).

9. Boldrin, D. et al. Giant piezomagnetism in $\mathrm{mn}_{3}$ nin. ACS Appl. Mater. Interfaces 10, 18863-18868 (2018).

10. Lin, J. et al. Giant negative thermal expansion covering room temperature in nanocrystalline gan $\mathrm{mn}_{3}$. Appl. Phys. Lett. 107, 131902-131906 (2015).

11. Takenaka, K. et al. Magnetovolume effects in manganese nitrides with antiperovskite structure. Sci. Technol. Adv. Mater. 15, 015009-015019 (2014).

12. Takenaka, K. \& Takagi, H. Giant negative thermal expansion in ge-doped antiperovskite manganese nitrides. Appl. Phys. Lett. 87, 261902-261904 (2005).

13. Matsunami, D., Fujita, A., Takenaka, K. \& Kano, M. Giant barocaloric effect enhanced by the frustration of the antiferromagnetic phase in $\mathrm{mn}_{3} \mathrm{gan}$. Nat Mater. 14, 73-78 (2015).

14. Peng, T., Bo-Sen, W. \& Yu-Ping, S. Mn-based antiperovskite functional materials: review of research. Chinese Phys. B 22, 067501-067512 (2013).

15. Shi, K. et al. Baromagnetic effect in antiperovskite $\mathrm{mn}_{3} \mathrm{ga}_{0.95} \mathrm{n}_{0.94}$ by neutron powder diffraction analysis. Adv. Mater. 28, 3761-3767 (2016).

16. Tohei, T., Wada, H. \& Kanomata, T. Negative magnetocaloric effect at the antiferromagnetic to ferromagnetic transition of $\mathrm{mn}_{3}$ gac. J. Appl. Phys. 94, 1800-1802 (2003).

17. Boldrin, D. et al. Multisite exchange-enhanced barocaloric response in $\mathrm{mn}_{3} \mathrm{nin}$ Phys. Rev. X 8, $041035-041041$ (2018).

18. Wang, B. et al. Large magnetic entropy change near room temperature in antiperovskite $\mathrm{sncmn}_{3}$. EPL 85, 47004-47008 (2009).

19. He, T. et al. Superconductivity in the non-oxide perovskite $\mathrm{mgcni}_{3}$. Nature $\mathbf{4 1 1}$ 54-56 (2001).

20. Lin, S. et al. Good thermoelectric performance in strongly correlated system sncCO $_{3}$ with antiperovskite structure. Inorg. Chem. 53, 3709-3715 (2014).

21. Shibayama, T. \& Takenaka, K. Giant magnetostriction in antiperovskite $\mathrm{mn}_{3} \mathrm{cun}$. J. Appl. Phys. 109, 07A928-07A930 (2011).

22. Chen, H., Niu, Q. \& MacDonald, A. H. Anomalous Hall effect arising from noncollinear antiferromagnetism. Phys. Rev. Lett. 112, 017205-017209 (2014).

23. Jungwirth, T., Marti, X., Wadley, P. \& Wunderlich, J. Antiferromagnetic spintronics. Nat. Nanotechnol. 11, 231-241 (2016).

24. Baltz, V. et al. Antiferromagnetic spintronics. Rev. Mod. Phys. 90, 015005-015061 (2018).

25. Žutić, l., Fabian, J. \& Das Sarma, S. Spintronics: fundamentals and applications. Rev. Mod. Phys. 76, 323-410 (2004).

26. Liu, E. et al. Giant anomalous Hall effect in a ferromagnetic kagome-lattice semimetal. Nat. Phys. 14, 1125-1131 (2018). 
27. Xu, G., Lian, B. \& Zhang, S.-C. Intrinsic quantum anomalous Hall effect in the kagome lattice $\mathrm{Cs}_{2} \operatorname{limn}_{3} \mathrm{f}_{12}$. Phys. Rev. Lett. 115, 186802-186805 (2015).

28. Wang, Q., Sun, S., Zhang, X., Pang, F. \& Lei, H. Anomalous Hall effect in a ferromagnetic $\mathrm{fe}_{3} \mathrm{Sn}_{2}$ single crystal with a geometrically frustrated fe bilayer kagome lattice. Phys. Rev. B 94, 075135-075139 (2016).

29. Gurung, G., Shao, D.-F., Paudel, T. R. \& Tsymbal, E. Y. Anomalous Hall conductivity of noncollinear magnetic antiperovskites. Phys. Rev. Mater. 3, $044409-044416$ (2019).

30. Samathrakis, I. \& Zhang, H. Tailoring the anomalous Hall effect in the noncollinear antiperovskite $\mathrm{mn}_{3}$ gan. Phys. Rev. B 101, 214423-214428 (2020).

31. Boldrin, D. et al. Anomalous Hall effect in noncollinear antiferromagnetic $\mathrm{mn}_{3} \mathrm{nin}$ thin films. Phys. Rev. Mater. 3, 094409-094415 (2019).

32. Zhou, X. et al. Spin-order dependent anomalous Hall effect and magneto-optical effect in the noncollinear antiferromagnets $\mathrm{mn}_{3} \mathrm{xn}$ with $\mathrm{x}=\mathrm{ga}, \mathrm{zn}$, ag, or ni. Phys. Rev. B 99, 104428-104440 (2019).

33. Gallego, S. V., Etxebarria, J., Elcoro, L., Tasci, E. S. \& Perez-Mato, J. M. Automatic calculation of symmetry-adapted tensors in magnetic and non-magnetic materials: a new tool of the bilbao crystallographic server. Acta Crystallogr A Found Adv. 75, 438-447 (2019).

34. Guo, G.-Y. \& Wang, T.-C. Large anomalous nernst and spin nernst effects in the noncollinear antiferromagnets $\mathrm{mn}_{3} \mathrm{x}(\mathrm{x}=\mathrm{sn}$, ge, ga). Phys. Rev. $B$ 96, 224415-224423 (2017).

35. Kübler, J. \& Felser, C. Non-collinear antiferromagnets and the anomalous Hall effect. EPL 108, 67001-67005 (2014).

36. Nayak, A. K. et al. Large anomalous Hall effect driven by a nonvanishing berry curvature in the noncolinear antiferromagnet $\mathrm{mn}_{3} g$. Sci. Adv. 2, e1501870-e1501874 (2016).

37. Bauer, G. E., Saitoh, E. \& Van Wees, B. J. Spin caloritronics. Nat. Mater. 11, 391-399 (2012).

38. Boona, S. R., Myers, R. C. \& Heremans, J. P. Spin caloritronics. Energy Environ. Sci. 7, 885-910 (2014).

39. Zhou, X. et al. Giant anomalous nernst effect in noncollinear antiferromagnetic mn-based antiperovskite nitrides. Phys. Rev. Mater. 4, 024408-024415 (2020).

40. Sakai, A. et al. Giant anomalous nernst effect and quantum-critical scaling in a ferromagnetic semimetal. Nat. Phys. 14, 1119-1124 (2018).

41. Guin, S. N. et al. Anomalous nernst effect beyond the magnetization scaling relation in the ferromagnetic heusler compound $\mathrm{CO}_{2}$ mnga. NPG Asia Mater. 11, 11-16 (2019).

42. Singh, H. K. et al. High-throughput screening of magnetic antiperovskites. Chem. Mater. 30, 6983-6991 (2018).

43. Liu, Z. et al. Antiferromagnetic piezospintronics. Adv. Electron. Mater. 5, 1900176-1900184 (2019).

44. Bouchaud, J.-P. et al. Antiferromagnetic-ferromagnetic transition in the compound mn3gac. J. Appl. Phys. 37, 971-972 (1966).

45. Kenmotsu, A., Shinohara, T. \& Watanabe, H. Nuclear magnetic resonance of ferromagnetic mn3alc and mn3gac. J. Phys. Soc. Jpn. 32, 377-381 (1972).

46. Kanomata, T., Kaneko, T. \& Nakagawa, Y. Magnetic properties of the intermetallic compound mn3inc. J. Solid State Chem. 96, 451-454 (1992).

47. Fruchart, D. \& F. Bertaut, E. Magnetic studies of the metallic perovskite-type compounds of manganese. J. Phys. Soc. Jpn. 44, 781-791 (1978).

48. Fruchart, D., Bertaut, E., Senateur, J. \& Fruchart, R. Magnetic studies on the metallic perovskite-type compound $\mathrm{mn}_{3} \mathrm{snn}$. J. Phys. Lett. 38, 21-23 (1977).

49. Deng, $S$. et al. Frustrated triangular magnetic structures of $m_{3} z n n$ : applications in thermal expansion. J. Phys. Chem. C 119, 24983-24990 (2015).

50. Huyen, V. T. N., Suzuki, M.-T., Yamauchi, K. \& Oguchi, T. Topology analysis for anomalous Hall effect in the noncollinear antiferromagnetic states of $\mathrm{mn}_{3}$ an $(\mathrm{a}=$ ni, cu, zn, ga, ge, pd, in, sn, ir, pt). Phys. Rev. B 100, 094426-094434 (2019).

51. Giannozzi, P. et al. Quantum Espresso: a modular and open-source software project for quantum simulations of materials. J. Phys. Condens. Matter. 21, 395502-395520 (2009).

52. Antropov, V., Ke, L. \& Åberg, D. Constituents of magnetic anisotropy and a screening of spin-orbit coupling in solids. Solid State Commun. 194, 35-38 (2014).

53. Buschow, K. H. J. Handbook of Magnetic Materials, Vol. 13 (Elsevier, 2003).

54. Abrikosov, I. A., Ponomareva, A., Steneteg, P., Barannikova, S. \& Alling, B. Recent progress in simulations of the paramagnetic state of magnetic materials. Curr. Opin. Solid State Mater. Sci. 20, 85-106 (2016).

55. Bocarsly, J. D. et al. A simple computational proxy for screening magnetocaloric compounds. Chem. Mater. 29, 1613-1622 (2017).

56. Date, M., Kanamori, J. \& Tachiki, M. Origin of magnetoelectric effect in $\mathrm{Cr}_{2} \mathrm{O}_{3}$. J. Phys. Soc. Jpn. 16, 2589-2589 (1961).

57. Ryu, J., Priya, S., Uchino, K. \& Kim, H.-E. Magnetoelectric effect in composites of magnetostrictive and piezoelectric materials. J. Eelectroceram. 8, 107-119 (2002).

58. Tashiro, H., Suzuki, R., Miyawaki, T., Ueda, K. \& Asano, H. Preparation and properties of inverse perovskite $\mathrm{mn}_{3}$ gan thin films and heterostructures. J. Korean Phys. Soc. 63, 299-301 (2013).
59. Nagaosa, N., Sinova, J., Onoda, S., MacDonald, A. H. \& Ong, N. P. Anomalous Hall effect. Rev. Mod. Phys. 82, 1539-1592 (2010).

60. Xiao, D., Yao, Y., Fang, Z. \& Niu, Q. Berry-phase effect in anomalous thermoelectric transport. Phys. Rev. Lett. 97, 026603-026606 (2006).

61. Noky, J., Gooth, J., Felser, C. \& Sun, Y. Characterization of topological band structures away from the fermi level by the anomalous nernst effect. Phys. Rev. $B$ 98, 241106-241110 (2018).

62. Sakai, A. et al. Iron-based binary ferromagnets for transverse thermoelectric conversion. Nature 581, 53-57 (2020).

63. Kresse, G. \& Furthmüller, J. Efficient iterative schemes for ab initio total-energy calculations using a plane-wave basis set. Phys. Rev. B 54, 11169-11186 (1996).

64. Perdew, J. P., Burke, K. \& Ernzerhof, M. Generalized gradient approximation made simple. Phys. Rev. Lett. 77, 3865-3868 (1996).

65. Zunger, A., Wei, S.-H., Ferreira, L. G. \& Bernard, J. E. Special quasirandom structures. Phys. Rev. Lett. 65, 353-356 (1990).

66. van de Walle, A., Asta, M. D. \& Ceder, G. The Alloy Theoretic Automated Toolkit: a user guide. Calphad 26, 539-553 (2002).

67. Stockem, I. et al. Anomalous phonon lifetime shortening in paramagnetic crn caused by spin-lattice coupling: a combined spin and ab initio molecular dynamics study. Phys. Rev. Lett. 121, 125902-125907 (2018).

68. Wu, Q., Zhang, S., Song, H.-F., Troyer, M. \& Soluyanov, A. A. Wanniertools: an open-source software package for novel topological materials. Comput. Phys. Commun. 224, 405-416 (2018).

69. Mostofi, A. A. et al. wannier90: a tool for obtaining maximally-localised wannier functions. Comput. Phys. Commun. 178, 685-699 (2008).

70. Xiao, D., Chang, M.-C. \& Niu, Q. Berry phase effects on electronic properties. Rev. Mod. Phys. 82, 1959-2007 (2010).

71. Bertaut, E., Fruchart, D., Bouchaud, J. \& Fruchart, R. Diffraction neutronique de $\mathrm{mn}_{3}$ gan. Solid State Commun. 6, 251-256 (1968).

\section{ACKNOWLEDGEMENTS}

The authors are grateful and acknowledge TU Darmstadt Lichtenberg highperformance computer support for the computational resources where the calculations were conducted for this project. The authors thank Prof. Manuel Richter of IFW Dresden for providing the SOC strength data and discussion. This project was supported by the Deutsche Forschungsgemeinschaft (DFG, German Research Foundation) Project-ID 405553726-TRR 270. N.M.F. acknowledges European Research Council (ERC) funding for financial support under the European Union's Horizon 2020 research and innovation programme (Grant No. 743116 project Cool Innov). The work of J.Z. was supported by the Ministry of Education, Youth and Sports of the Czech Republic from the OP RDE program under the project International Mobility of Researchers MSCA-IF at CTU No. CZ.02.2.69/0.0/0.0/18_070/0010-457.

\section{AUTHOR CONTRIBUTIONS}

H.K.S. preformed all the calculations. All authors discussed the results. The manuscript was written by H.K.S. All authors reviewed and approved the manuscript.

\section{FUNDING}

Open Access funding enabled and organized by Projekt DEAL.

\section{COMPETING INTERESTS}

The authors declare no competing interests.

\section{ADDITIONAL INFORMATION}

Supplementary information The online version contains supplementary material available at https://doi.org/10.1038/s41524-021-00566-w.

Correspondence and requests for materials should be addressed to H.K.S. or H.Z.

Reprints and permission information is available at http://www.nature.com/ reprints

Publisher's note Springer Nature remains neutral with regard to jurisdictional claims in published maps and institutional affiliations. 
Open Access This article is licensed under a Creative Commons Attribution 4.0 International License, which permits use, sharing, adaptation, distribution and reproduction in any medium or format, as long as you give appropriate credit to the original author(s) and the source, provide a link to the Creative Commons license, and indicate if changes were made. The images or other third party material in this article are included in the article's Creative Commons license, unless indicated otherwise in a credit line to the material. If material is not included in the article's Creative Commons license and your intended use is not permitted by statutory regulation or exceeds the permitted use, you will need to obtain permission directly from the copyright holder. To view a copy of this license, visit http://creativecommons. org/licenses/by/4.0/.

(c) The Author(s) 2021 